K. Kato

Nagoya Math. J.

Vol. 69 (1978), 121-129

\title{
STOCHASTIC STABILITY OF ANOSOV DIFFEOMORPHISMS
}

\author{
KAZUHISA KATO
}

\section{§ 0. Introduction}

R. Bowen [1] introduced the notion of pseudo-orbit for a homeomorphism $f$ of a metric space $X$ as follows: A (double) sequence $\left\{x_{i}\right\}_{i \in Z}$ of points $x_{i}$ in $X$ is called a $\delta$-pseudo-orbit of $f$ iff

$$
d\left(f x_{i}, x_{i+1}\right) \leq \delta
$$

for every $i \in Z$, where $d$ denotes the metric in $X$. We say $f$ is stochastically stable if for every $\varepsilon>0$ there exists $\delta>0$ such that every $\delta$ pseudo-orbit $\left\{x_{i}\right\}_{i \in Z}$ of $f$ is $\varepsilon$-traced by some $x \in X$, i.e.,

$$
d\left(f^{i} x, x_{i}\right) \leq \varepsilon
$$

for every $i \in \boldsymbol{Z}$. He proved in [1] that if a compact hyperbolic set $\Lambda$ for a diffeomorphism $f$ of a compact manifold $M$ has local product structure then the restriction $f \mid \Lambda$ of $f$ to $\Lambda$ is stochastically stable, using stable and unstable manifolds.

In this paper we prove first that an Anosov diffeomorphism $f$ of a compact manifold $M$ is topologically stable, in the set of all continuous maps of $M$ into $M$, in a sense (Theorem 1). Next, making use of Theorem 1 we give another proof for Bowen's result, in the case of $f$ an Anosov diffeomorphism (Theorem 2). The idea of this paper is inspired by a result of $\mathrm{A}$. Morimoto [2], which says that a topologically stable homeomorphism $f$ of a manifold $M$ with $\operatorname{dim} M \geq 3$ is stochastically stable. The method of the proof follows that of P. Walters [3].

The author would like to express his gratitude to Professor A. Morimoto for several useful conversations and his advices.

\section{§1. Preparatory lemmas}

$M$ will always denote a compact $C^{\infty}$ manifold without boundary.

Received February 18, 1977. 
Definition 1. A $C^{1}$ diffeomorphism $f$ of $M$ is called an Anosov diffeomorphism if there exist a Riemannian metric $\|\cdot\|$ on $M$ and constants $C>0,0<\lambda<1$ such that the tangent bundle of $M$ can be written as the Whitney sum of two continuous subbundles, $T M=E^{s} \oplus E^{u}$, and the following conditions are satisfied:

$$
\begin{array}{cl}
T f\left(E^{\sigma}\right)=E^{\sigma} & (\sigma=s, u) . \\
\left\|T f^{n}(v)\right\| \leq C \lambda^{n}\|v\|, & v \in E^{s}, n \geq 0, \\
\left\|T f^{-n}(v)\right\| \leq C \lambda^{n}\|v\|, & v \in E^{u}, n \geq 0 .
\end{array}
$$

$f$ will always denote an Anosov diffeomorphism of $M$. We can find a Riemannian metric for which we can take $C=1$, and fix it (cf. [3]). Let $\mathfrak{X}(M)$ denote the Banach space of all continuous vector fields with the norm

$$
\|v\|=\sup _{x \in M}\|v(x)\|, \quad v \in \mathfrak{X}(M) .
$$

Let $\mathfrak{X}^{\sigma}(M)$ denote the subspace of all $v \in \mathfrak{X}(M)$ with $v(x) \in E_{x}^{\sigma}$ for every $x \in M(\sigma=s, u)$. Clearly $\mathfrak{X}(M)=\mathfrak{X}^{s}(M) \oplus \mathfrak{X}^{u}(M)$ (direct sum). We define a linear operator $f_{\#}: \mathfrak{X}(M) \rightarrow \mathfrak{X}(M)$ by

$$
f_{\#}(v)=T f \circ v \circ f^{-1}, \quad v \in \mathfrak{X}(M) .
$$

Let $d\left(\right.$, ) denote the metric on $M$ induced by $\|\cdot\|$, and for each $x \in M \exp _{x}$ : $T M_{x} \rightarrow M$ denote the exponential map with respect to $\|\cdot\|$. Let $\operatorname{Map}(M)$ denote the metric space of all continuous maps of $M$ into $M$ with the metric

$$
d(\phi, \psi)=\sup _{x \in M} d(\phi x, \psi x), \quad \phi, \psi \in \operatorname{Map}(M)
$$

For $\delta>0$ we put $\operatorname{Map}(M, \delta)=\{\phi \in \operatorname{Map}(M): d(\phi, \mathrm{id}) \leq \delta\}$, and $\sum_{\delta}=$ $\{(x, y) \in M \times M: d(x, y) \leq \delta\}$.

The following lemma is due to P. Walters [3].

LEMMA 1. There exist $\delta_{1}>0$ and $\tau_{1}>0$ satisfying the following conditions :

(1.3) For every $(x, y) \in \sum_{\delta_{1}}$ there exists a linear isomorphism $L_{(x, y)}: T M_{x}$ $\rightarrow T M_{y}$ such that $L_{(x, y)}\left(E_{x}^{\sigma}\right)=E_{y}^{o}(\sigma=s, u)$, and $L_{(x, y)}$ is continuous with respect to $(x, y) \in \sum_{\delta_{1}}$.

(1.4) For every $(x, y) \in \sum_{\delta_{1}}$ there exists a continuous $\operatorname{map} \gamma_{(x, y)}: T M_{x}\left(\tau_{1}\right)$ $\rightarrow T M_{y}$ such that 


$$
\exp _{x}(v)=\exp _{y}\left(L_{(x, y)}(v)+\gamma_{(x, y)}(v)\right), \quad v \in T M_{x}\left(\tau_{1}\right)
$$

and $\gamma_{(x, y)}$ is continuous with respect to $(x, y) \in \sum_{\delta_{1}}$, where $T M_{x}\left(\tau_{1}\right)=$ $\left\{v \in T M_{x}:\|v\| \leq \tau_{1}\right\}$.

(1.5) $x=\exp _{y}\left(\gamma_{(x, y)}(0)\right), \quad(x, y) \in \sum_{\delta_{1}}$.

(1.6) $\left\|L_{(x, y)}\right\|$ and $\left\|\left(L_{(x, y)}\right)^{-1}\right\|$ converge uniformly to 1 as $d(x, y) \rightarrow 0$.

(1.7) For every $(x, y) \in \sum_{\delta_{1}}$ there exists $K(x, y) \geq 0$ such that

$$
\left\|\gamma_{(x, y)}(v)-\gamma_{(x, y)}\left(v^{\prime}\right)\right\| \leq K(x, y)\left\|v-v^{\prime}\right\|, \quad v, v^{\prime} \in T M_{x}\left(\tau_{1}\right)
$$

and $K(x, y)$ converges uniformly to 0 as $d(x, y) \rightarrow 0$.

Proof. See Lemma 1 [3].

Definition 2. For $\phi \in \operatorname{Map}\left(M, \delta_{1}\right)$ we define continuous linear maps $J_{\phi}, R_{\phi}: \mathfrak{X}(M) \rightarrow \mathfrak{X}(M)$, a continuous map $\gamma_{\phi}: \mathfrak{X}(M)\left(\tau_{1}\right) \rightarrow \mathfrak{X}(M)$, and a constant $K(\phi) \geq 0$ as follows: For $v \in \mathfrak{X}(M)$ and $x \in M$

$$
\begin{aligned}
J_{\phi}(v)(x) & =L_{(\phi x, x)}(v(\phi x)), \\
R_{\phi}(v)(x) & =\left(L_{(x, \phi x)}\right)^{-1}(v(\phi x)) .
\end{aligned}
$$

For $v \in \mathfrak{X}(M)\left(\tau_{1}\right)$ and $x \in M$

$$
\gamma_{\phi}(v)(x)=\gamma_{(\phi x, x)}(v(\phi x))
$$

where $\mathfrak{X}(M)\left(\tau_{1}\right)=\left\{v \in \mathfrak{X}(M):\|v\| \leq \tau_{1}\right\}$.

$$
K(\phi)=\sup _{x \in M} K(\phi x, x) \text {. }
$$

By Lemma 1 we have the following lemma:

Lemma 2. For $\phi \in \operatorname{Map}\left(M, \delta_{1}\right), v, v^{\prime} \in \mathfrak{X}(M)\left(\tau_{1}\right)$ and $x \in M$

$$
\begin{gathered}
J_{\phi}\left(\mathfrak{X}^{\sigma}(M)\right) \subset \mathfrak{X}^{\sigma}(M), R_{\phi}\left(\mathfrak{X}^{\sigma}(M)\right) \subset \mathfrak{X}^{\sigma}(M) \quad(\sigma=s, u), \\
\exp _{\phi x} v(\phi x)=\exp _{x}\left(J_{\phi}(v)+\gamma_{\phi}(v)\right)(x), \\
\exp _{x} \gamma_{\phi}(0)=\phi(x), \\
\left\|\gamma_{\phi}(v)-\gamma_{\phi}\left(v^{\prime}\right)\right\| \leq K(\phi)\left\|v-v^{\prime}\right\| \\
K(\phi) \longrightarrow 0 \text { as } d(\phi, \mathrm{id}) \longrightarrow 0 \\
\left\|J_{\phi}\right\|,\left\|R_{\phi}\right\| \longrightarrow 1 \text { as } d(\phi, \mathrm{id}) \longrightarrow 0
\end{gathered}
$$

LeMma 3. If $\phi, \psi \in \operatorname{Map}\left(M, \delta_{1}\right)$ and a subset $S$ of $M$ satisfy 


$$
\psi \phi(x)=x
$$

for every $x \in S$, then

$$
\begin{aligned}
R_{\psi} J_{\dot{\phi}}(v)(\phi x) & =v(\phi x), \\
J_{\phi} R_{\psi}(v)(x) & =v(x)
\end{aligned}
$$

for every $x \in S$ and $v \in \mathfrak{X}(M)$.

Proof. By Definition 2 we have

$$
\begin{aligned}
J_{\dot{\phi}} R_{\psi}(v)(x) & =L_{(\phi x, x)}\left(R_{\psi}(v)(\phi x)\right) \\
& =L_{(\phi x, x)}\left(L_{(\dot{\phi} x, \psi \phi x)}\right)^{-1}(v(\psi \phi x)) \\
& =v(x),
\end{aligned}
$$

which proves (1.14). Similarly, we have

$$
\begin{aligned}
R_{\psi} J_{\dot{\phi}}(v)(\phi x) & =\left(L_{(\dot{\phi} x, \psi \dot{\phi} x)}\right)^{-1}\left(J_{\dot{\phi}}(v)(\psi \dot{\phi} x)\right) \\
& =\left(L_{(\dot{\phi} x, x)}\right)^{-1} L_{(\phi x, x)}(v(\phi x)) \\
& =v(\phi x),
\end{aligned}
$$

which proves (1.13).

LEMMA 4. There exists $\tau_{2}>0$ satisfying the following conditions: For every $v \in \mathfrak{X}(M)\left(\tau_{2}\right)$ there exists $s(v) \in \mathfrak{X}(M)$ such that

$$
\begin{aligned}
& f \exp _{f-1 x} v\left(f^{-1} x\right)=\exp _{x}\left(f_{\sharp}(v)+s(v)\right)(x), \quad x \in M, \\
& s(0)=0,
\end{aligned}
$$

$$
\left\|s(v)-s\left(v^{\prime}\right)\right\| \leq C\left(\tau_{2}\right)\left\|v-v^{\prime}\right\|
$$

for every $v$, $v^{\prime} \in \mathfrak{X}(M)\left(\tau_{2}\right)$, where $C\left(\tau_{2}\right) \rightarrow 0$ as $\tau_{2} \rightarrow 0$.

Proof. See Lemma 2 [3].

LEMmA 5. There exist constants $0<\delta_{2}<\delta_{1}$ and $\alpha>0$ satisfying the following conditions: For every $\phi, \downarrow \in \operatorname{Map}\left(M, \delta_{2}\right)$ there exist a constant $\mu(\phi, \psi)>0$ and a continuous linear map $P=P_{\phi, \psi}: \mathfrak{X}(M) \rightarrow \mathfrak{X}(M)$ such that if a subset $S$ of $M$ satisfies

$$
\psi \phi(x)=x
$$

for every $x \in S$, then

$$
\left(I-R_{\psi} f_{\#}\right) P(v)(\phi x)=v(\phi x)
$$


for every $x \in S$, and

$$
\begin{gathered}
\|P\| \leq \frac{\alpha}{1-\mu(\phi, \psi) \lambda}, \\
\mu(\phi, \psi) \longrightarrow 1 \text { as } d(\phi, \mathrm{id}), d(\psi, \mathrm{id}) \longrightarrow 0 .
\end{gathered}
$$

Proof. There exists $\alpha>0$ such that

$$
\left\|v_{s}\right\|+\left\|v_{u}\right\| \leq \alpha\left\|v_{s}+v_{u}\right\|
$$

for every $v_{\sigma} \in \mathfrak{X}^{\sigma}(M)(\sigma=s, u)$. For $\phi, \psi \in \operatorname{Map}\left(M, \delta_{1}\right)$ we put

$$
\mu(\phi, \psi)=\operatorname{Max}\left\{\left\|J_{\phi}\right\|,\left\|R_{\psi}\right\|\right\} \text {. }
$$

Then, by (1.12) there exists $0<\delta_{2} \leq \delta_{1}$ and $\lambda_{1}$ such that

$$
\mu(\phi, \psi) \lambda<\lambda_{1}<1
$$

for every $\phi, \psi \in \operatorname{Map}\left(M, \delta_{2}\right)$.

By (1.1) and (1.8) we can define as follows: $f_{\sharp}^{o}=f_{\sharp} \mid \mathfrak{X}^{o}(M), J_{\phi}^{o}=$ $J_{\phi} \mid \mathfrak{X}^{\sigma}(M)$ and $R_{\psi}^{\sigma}=R_{\psi} \mid \mathfrak{X}^{\sigma}(M)(\sigma=s, u)$. By (1.2), (1.20) and (1.21) we have

$$
\left\|R_{\psi}^{s} f_{\sharp}^{s}\right\| \leq\left\|R_{\psi}^{s}\right\|\left\|f_{\#}^{s}\right\| \leq \mu(\phi, \psi) \lambda<1 .
$$

Therefore, the Neumann series $\sum_{n=0}^{\infty}\left(R_{\psi}^{s} f_{\sharp}^{s}\right)^{n}$ is convergent. Putting $P_{s}$ $=\sum_{n=0}^{\infty}\left(R_{\psi}^{s} f_{\sharp}^{s}\right)^{n}$ we have

$$
\left\|P_{s}\right\| \leq \frac{1}{1-\mu(\phi, \psi) \lambda} .
$$

Similarly, since $\left\|\left(f_{\#}^{u}\right)^{-1} J_{\phi}^{u}\right\| \leq \mu(\phi, \psi) \lambda<1$ the Neumann series $\sum_{n=1}^{\infty}\left(\left(f_{\#}^{u}\right)^{-1} J_{\phi}^{u}\right)^{n}$ is convergent. Putting $P_{u}=-\sum_{n=1}^{\infty}\left(\left(f_{\sharp}^{u}\right)^{-1} J_{\phi}^{u}\right)^{n}$ we have

$$
\left\|P_{u}\right\| \leq \frac{1}{1-\mu(\phi, \psi) \lambda} \text {. }
$$

Now we put $P=P_{s}+P_{u}$. By (1.19), (1.22) and (1.23) we get

$$
\|P\| \leq \alpha \operatorname{Max}\left\{\left\|P_{s}\right\|,\left\|P_{u}\right\|\right\} \leq \frac{\alpha}{1-\mu(\phi, \psi) \lambda},
$$

which proves (1.18). Next, we shall prove (1.17). By (1.13) and (1.14) we have

$$
\begin{aligned}
(I- & \left.R_{\psi}^{u} f_{\#}^{u}\right) P_{u}(v)(\phi x) \\
& =P_{u}(v)(\phi x)+R_{\psi}^{u} f_{\#}^{u}\left(f_{\#}^{u}\right)^{-1} J_{\phi}^{u}\left[\sum_{n=0}^{\infty}\left(\left(f_{\#}^{u}\right)^{-1} J_{\phi}^{u}\right)^{n}(v)\right](\phi x) \\
& =P_{u}(v)(\phi x)+\sum_{n=0}^{\infty}\left(\left(f_{\sharp}^{u}\right)^{-1} J_{\phi}^{u}\right)^{n}(v)(\phi x) \\
& =v(\phi x)
\end{aligned}
$$


for $v \in \mathfrak{X}^{u}(M)$ and $x \in S$. Clearly, $\left(I-R_{\psi}^{s} f_{\sharp}^{s}\right) P_{s}=I$.

Thus, we have proved (1.17).

\section{$\S 2$. Proof of Theorem 1}

THEOREM 1. An Anosov diffeomorphism $f$ of $M$ is topologically stable in the following sense: For every $\varepsilon>0$ there exists $\delta=\delta(\varepsilon)>0$ satisfying the following conditions: If $g, \tilde{g} \in \operatorname{Map}(M)$ with $d(f, g)$, d(f $\tilde{g}, \mathrm{id})$ $\leq \delta$ and $a$ subset $S$ of $M$ satisfy

$$
\tilde{g} g(x)=x
$$

for every $x \in S$, then there exists $h \in \operatorname{Map}(M)$ such that

$$
h g(x)=f h(x)
$$

for every $x \in S$, and

$$
d(h, \mathrm{id}) \leq \varepsilon
$$

Proof. First, take $\varepsilon_{0} \leq \operatorname{Min}\left\{\tau_{1}, \tau_{2}, \varepsilon\right\}$ so small that for every $\phi$, $\psi \in \operatorname{Map}\left(M, \delta_{2}\right)$

$$
\frac{\alpha \mu(\phi, \psi)}{1-\mu(\phi, \psi) \lambda} C\left(\varepsilon_{0}\right) \leq \frac{1}{4}
$$

This is possible since $C\left(\varepsilon_{0}\right) \rightarrow 0$ as $\varepsilon_{0} \rightarrow 0$. Next, take $0<\delta \leq \delta_{2}$ so small that for every $\phi, \psi \in \operatorname{Map}(M, \delta)$

$$
\frac{\alpha \mu(\phi, \psi)}{1-\mu(\phi, \psi) \lambda} \delta \leq \frac{1}{2} \varepsilon_{0}
$$

and

$$
\frac{\alpha \mu(\phi, \psi)}{1-\mu(\phi, \psi) \lambda} K(\phi) \leq \frac{1}{4} .
$$

This is possible since $K(\phi) \rightarrow 0$ as $d(\phi, \mathrm{id}) \rightarrow 0$.

For $\phi, \psi \in \operatorname{Map}(M, \delta)$ we define a continuous map $\Phi: \mathfrak{X}(M)\left(\varepsilon_{0}\right) \rightarrow \mathfrak{X}(M)$ by

$$
\Phi(v)=P_{\phi, \psi} R_{\psi}\left(s(v)-\gamma_{\phi}(v)\right), \quad v \in \mathfrak{X}(M)\left(\varepsilon_{0}\right) .
$$

To find a fixed point of $\Phi$ we shall first show that the Lipschitz constant of $\Phi \leq \frac{1}{2}$. Take two elements $v, v^{\prime} \in \mathfrak{X}(M)\left(\varepsilon_{0}\right)$. By (1.11), (1.16), (1.18), (1.20), (2.3) and (2.5) we have 


$$
\begin{aligned}
\| \Phi(v) & -\Phi\left(v^{\prime}\right) \| \\
& \leq\|P\|\left\|R_{\psi}\right\|\left(\left\|s(v)-s\left(v^{\prime}\right)\right\|+\left\|\gamma_{\phi}(v)-\gamma_{\phi}\left(v^{\prime}\right)\right\|\right) \\
& \leq \frac{\alpha \mu(\phi, \psi)}{1-\mu(\phi, \psi) \lambda}\left(C\left(\varepsilon_{0}\right)\left\|v-v^{\prime}\right\|+K(\phi)\left\|v-v^{\prime}\right\|\right) \\
& \leq\left(\frac{1}{4}+\frac{1}{4}\right)\left\|v-v^{\prime}\right\|=\frac{1}{2}\left\|v-v^{\prime}\right\| .
\end{aligned}
$$

Next, we shall show $\Phi\left(\mathfrak{X}(M)\left(\varepsilon_{0}\right)\right) \subset \mathfrak{X}(M)\left(\varepsilon_{0}\right)$. By (1.10), (1.15), (1.18), (1.20) and (2.4) we have

$$
\begin{aligned}
\|\Phi(v)\| & \leq\|\Phi(0)\|+\|\Phi(v)-\Phi(0)\| \\
& \leq\|P\|\left\|R_{\psi}\right\| \delta+\frac{1}{2}\|v\| \\
& \leq \frac{\alpha \mu(\phi, \psi)}{1-\mu(\phi, \psi) \lambda} \delta+\frac{1}{2} \varepsilon_{0} \\
& \leq \frac{1}{2} \varepsilon_{0}+\frac{1}{2} \varepsilon_{0}=\varepsilon_{0}
\end{aligned}
$$

for $v \in \mathfrak{X}(M)\left(\varepsilon_{0}\right)$. Thus, $\Phi$ is a contraction of a complete metric space $\mathfrak{X}(M)\left(\varepsilon_{0}\right)$. Therefore, $\Phi$ has a unique fixed point $v_{0}=v_{0}(\phi, \psi) \in \mathfrak{X}(M)\left(\varepsilon_{0}\right)$, i.e.

$$
v_{0}=P_{\phi, \psi} R_{\psi}\left(s\left(v_{0}\right)-\gamma_{\phi}\left(v_{0}\right)\right) \text {. }
$$

We put $h\left(=h_{\phi, \psi}\right)=\exp v_{0}$.

Now assume that $g, \tilde{g} \in \operatorname{Map}(M)$ with $d(f, g), d(f \tilde{g}$, id $) \leq \delta$ and a subset $S$ of $M$ satisfy that $\tilde{g} g(x)=x$ for every $x \in S$. Putting $\phi=g f^{-1}$ and $\psi=f \tilde{g}$ we see that $\phi, \psi \in \operatorname{Map}(M, \delta)$ and $\psi \phi(f x)=f(x)$ for every $x \in S$. By Definition 2, (1.14), (1.17) and (2.6) we obtain

$$
\begin{aligned}
J_{\phi}\left(v_{0}\right) & (f x)-f_{\#}\left(v_{0}\right)(f x) \\
& =J_{\phi}\left(v_{0}\right)(f x)-J_{\phi} R_{\psi} f_{\#}\left(v_{0}\right)(f x) \\
& =J_{\phi}\left(I-R_{\psi} f_{\sharp}\right)\left(v_{0}\right)(f x) \\
& =J_{\phi}\left(I-R_{\psi} f_{\sharp}\right) P R_{\psi}\left(s\left(v_{0}\right)-\gamma_{\phi}\left(v_{0}\right)\right)(f x) \\
& =L_{(\phi f x, f x)}\left[\left(I-R_{\psi} f_{\#}\right) P R_{\psi}\left(s\left(v_{0}\right)-\gamma_{\phi}\left(v_{0}\right)\right)(\phi f x)\right] \\
& =L_{(\phi f x, f x)}\left[R_{\psi}\left(s\left(v_{0}\right)-\gamma_{\phi}\left(v_{0}\right)\right)(\phi f x)\right] \\
& =L_{(\phi f x, f x)}\left(L_{(\phi f x, \psi \phi f x)}\right)^{-1}\left(\left(s\left(v_{0}\right)-\gamma_{\phi}\left(v_{0}\right)\right)(\psi \phi f x)\right) \\
& =s\left(v_{0}\right)(f x)-\gamma_{\phi}\left(v_{0}\right)(f x)
\end{aligned}
$$

for every $x \in S$. Thus we have

$$
\left(J_{\phi}\left(v_{0}\right)+\gamma_{\phi}\left(v_{0}\right)\right)(f x)=\left(f_{\#}\left(v_{0}\right)+s\left(v_{0}\right)\right)(f x)
$$

for every $x \in S$. By (1.9), (1.15) and (2.7), for every $x \in S$ we have 


$$
\begin{aligned}
& h g(x)=\exp _{\phi f x} v_{0}(\phi f x) \\
& =\exp _{f x}\left(J_{\phi}\left(v_{0}\right)+\gamma_{\phi}\left(v_{0}\right)\right)(f x) \\
& =\exp _{f x}\left(f_{\#}\left(v_{0}\right)+s\left(v_{0}\right)\right)(f x) \\
& =f \exp _{f-1 f x} v_{0}\left(f^{-1} f x\right) \\
& =f h(x) \text {, }
\end{aligned}
$$

which proves (2.1). Clearly, $d(h, i d)=\left\|v_{0}\right\| \leq \varepsilon_{0} \leq \varepsilon$, which proves (2.2). This completes the proof of Theorem 1 .

Remark. Let $g \in \operatorname{Map}(M)$ be a homeomorphism of $M$ with $d(f, g) \leq \delta$. Clearly, we see that $d\left(f g^{-1}, \mathrm{id}\right) \leq \delta$ and $g^{-1} g(x)=x$ for every $x \in M$. By Theorem 1 there exists $h \in \operatorname{Map}(M, \varepsilon)$ such that

$$
h g(x)=f h(x)
$$

for every $x \in M$. Thus, Theorem 1 is a generalization of $\mathrm{P}$. Walters' result (Theorem 1 [3]), except the uniqueness of the semiconjugacy $h$ with $d(h, \mathrm{id}) \leq \varepsilon$.

\section{§3. Proof of Theorem 2}

THEOREM 2. An Anosov diffeomorphism $f$ of $M$ is stochastically stable.

Proof. For $\varepsilon>0$ we put $\delta_{0}=\delta(\varepsilon / 2)$, where $\delta(\varepsilon / 2)$ is as in Theorem 1 , and $\delta=\delta_{0} / 3$. For every $\delta$-pseudo-orbit $\left\{x_{i}\right\}_{i \in Z}$ of $f$, we shall find $x \in M$ such that

$$
d\left(f^{i} x, x_{i}\right) \leq \varepsilon, \quad i \in Z .
$$

ClaIM 1. For every positive integer $k$ and $\delta$-pseudo-orbit $\left\{x_{i}\right\}_{i \in Z}$ of $f$, there exists $z \in M$ such that

$$
d\left(f^{i} z, x_{i}\right) \leq \varepsilon, \quad i=0,1, \cdots, k .
$$

Proof. There exists a $\left(\frac{2}{3} \delta_{0}\right)$-pseudo-orbit $\left\{x_{i}^{\prime}\right\}_{i \in Z}$ such that

$$
\begin{array}{ll}
d\left(x_{i}^{\prime}, x_{i}\right) \leq \varepsilon / 2, & i=0,1, \cdots, k, \\
x_{i}^{\prime} \neq x_{j}^{\prime}, & 0 \leq i \neq j \leq k+1 .
\end{array}
$$

Since $f\left(x_{i}^{\prime}\right) \neq f\left(x_{j}^{\prime}\right)(0 \leq i \neq j \leq k+1)$ and $d\left(f x_{i}^{\prime}, x_{i+1}^{\prime}\right) \leq \frac{2}{3} \delta_{0}$, we can find $\phi, \psi \in \operatorname{Map}\left(M, \delta_{0}\right)$ such that

$$
\phi f\left(x_{i}^{\prime}\right)=x_{i+1}^{\prime}, \psi\left(x_{i+1}^{\prime}\right)=f\left(x_{i}^{\prime}\right), \quad i=0,1, \cdots, k .
$$


Put $S=\left\{x_{0}^{\prime}, \cdots, x_{k}^{\prime}\right\}, g=\phi f$ and $\tilde{g}=f^{-1} \psi$. Then we see that $d(f, g)=$ $d(\phi, \mathrm{id}), d(f \tilde{g}, \mathrm{id})=d(\psi, \mathrm{id}) \leq \delta_{0}$, and $\tilde{g} g\left(x_{i}^{\prime}\right)=f^{-1} \psi \phi f\left(x_{i}^{\prime}\right)=x_{i}^{\prime}, \quad i=0,1$, $\cdots, k$. By Theorem 1, there exists $h \in \operatorname{Map}(M, \varepsilon / 2)$ such that $h g\left(x_{i}^{\prime}\right)=$ $f h\left(x_{i}^{\prime}\right)$, for $i=0,1, \cdots, k$. Therefore, we have

$$
f^{i} h\left(x_{0}^{\prime}\right)=h\left(x_{i}^{\prime}\right), \quad i=0,1, \cdots, k .
$$

Putting $z=h\left(x_{0}^{\prime}\right)$, by (3.3) and (3.4) we obtain

$$
\begin{aligned}
d\left(f^{i} z, x_{i}\right) & \leq d\left(f^{i} h\left(x_{0}^{\prime}\right), x_{i}^{\prime}\right)+d\left(x_{i}^{\prime}, x_{i}\right) \\
& \leq d\left(h\left(x_{i}^{\prime}\right), x_{i}^{\prime}\right)+\varepsilon / 2 \\
& \leq d(h, \mathrm{id})+\varepsilon / 2 \\
& \leq \varepsilon / 2+\varepsilon / 2=\varepsilon
\end{aligned}
$$

which proves (3.2).

ClaIm 2. Let $\left\{x_{i}\right\}_{i \in Z}$ be a $\delta$-pseudo-orbit of $f$. For every positive integer $k$ there exists $z=z_{k} \in M$ such that

$$
d\left(f^{i} z, x_{i}\right) \leq \varepsilon, \quad|i| \leq k .
$$

Proof. Take a positive integer $k$ and fix it. Putting $y_{i}=x_{-k+i}$ we see that $\left\{y_{i}\right\}_{i \in Z}$ is a $\delta$-pseudo-orbit. By Claim 1 there exists $z^{\prime} \in M$ such that $d\left(f^{i} z^{\prime}, y_{i}\right) \leq \varepsilon$, for $i=0,1, \cdots, 2 k$. Putting $z=f^{k}\left(z^{\prime}\right)$ we get $d\left(f^{i} z, x_{i}\right)$ $=d\left(f^{i+k} z^{\prime}, y_{i+k}\right) \leq \varepsilon,|i| \leq k$, which proves (3.5).

By the compactness of $M$ we can find a subsequence $\left\{z_{k_{\nu}}\right\}$ of $\left\{z_{k}\right\}$ such that $\lim _{\nu \rightarrow \infty} z_{k_{\nu}}=x$ for some $x \in M$. Take $i \in Z$ and fix it. By (3.5) we have that $d\left(f^{i} z_{k_{\nu}}, x_{i}\right) \leq \varepsilon$ for every $\nu$ with $|i| \leq k_{\nu}$. Therefore we obtain $d\left(f^{i} x, x_{i}\right)=\lim _{\nu \rightarrow \infty} d\left(f^{i} z_{k_{\nu}}, x_{i}\right) \leq \varepsilon$, which proves $(3.1)$.

This completes the proof of Theorem 2 .

\section{REFERENCES}

[1] R. Bowen, $\omega$-limit sets for Axiom A diffeomorphisms, J. Diff. Eq. 18 (1975), 333339.

[2] A. Morimoto, Stochastically stable diffeomorphisms and Takens conjecture, to appear.

[ 3 ] P. Walters, Anosov diffeomorphisms are topologically stable, Topology 9 (1970), $71-78$.

Kochi University 